04

\title{
Солнечный телескоп вакуумного ультрафиолетового диапазона
} для наноспутников

\author{
(C) С.В. Кузин, ${ }^{1,2}$ C.А. Богачев, ${ }^{3,4}$ Н.Ф. Ерхова, ${ }^{3}$ А.А. Перцов, ${ }^{2,3,4}$ И.П. Лобода, ${ }^{3}$ А.А. Рева, ${ }^{3}$ А.А. Холодилов, ${ }^{3}$ \\ А.С. Ульянов, ${ }^{3}$ А.С. Кириченко, ${ }^{2,3,4}$ И.В. Малышев, ${ }^{5,6}$ А.Е. Пестов, ${ }^{5,6}$ В.Н. Полковников, ${ }^{5}$ М.Н. Торопов, ${ }^{5,6}$ \\ Н.Н. Цыбин, ${ }^{5}$ Н.И. Чхало, ${ }^{5}$ В.А. Крюковский, ${ }^{7}$ В.Н. Горев, ${ }^{8}$ А.А. Дорошкин, ${ }^{8}$ А.М. Задорожный, \\ В.Ю. Прокопьев ${ }^{8}$
}

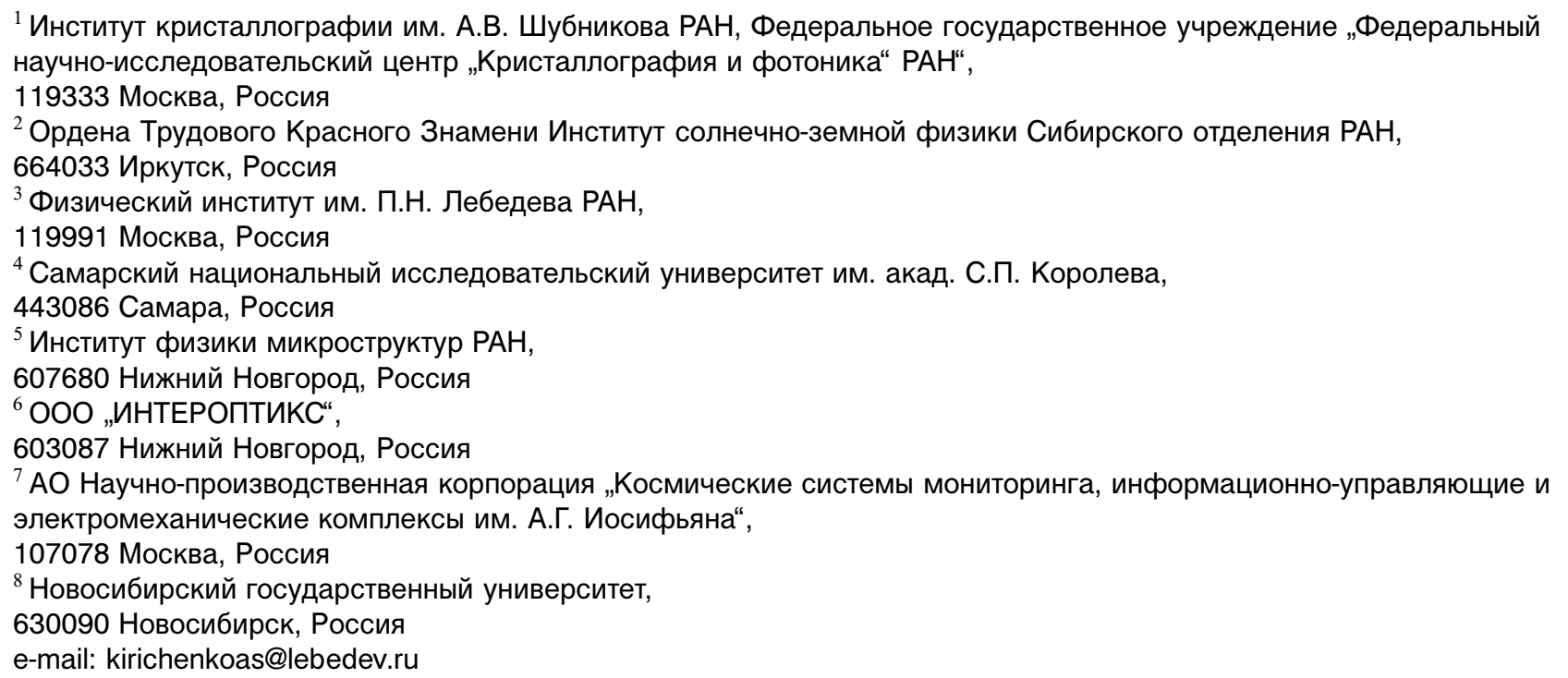

Поступило в Редакцию 21 апреля 2021 г.

В окончательной редакции 21 апреля 2021 г.

Принято к публикации 21 апреля 2021 г.

\begin{abstract}
В рамках программы „Универсат“ разработано семейство солнечных вакуумных ультрафиолетовых (ВУФ) телескопов для размещения на наноспутниках формата 6U. Телескопы предназначены для получения изображений солнечной короны, необходимых для мониторинга солнечной активности и выработки прогноза космической погоды. Обоснованы спектральные диапазоны наблюдений, оптимизированы характеристики наноспутника с точки зрения реализуемости наблюдений, рассчитана оптическая схема телескопа и состав многослойных зеркал ВУФ диапазона и тонкопленочных фильтров.
\end{abstract}

Ключевые слова: наноспутник, ВУФ-диапазон, телескоп, корона Солнца.

DOI: 10.21883/JTF.2021.10.51355.115-21

\section{Введение}

Несмотря на существенные ограничения по сравнению с полноразмерными космическими аппаратами, наноспутники становятся все более и более распространенной платформой для проведения прикладных и фундаментальных космических исследований. В области физики Солнца проведен ряд экспериментов по исследованию интегрального рентгеновского изучения короны в диапазоне $\sim 0.5-30 \mathrm{keV}$ (MinXSS [1], „Ярило“ [2]), которые показали возможность переноса части прикладных исследований на наноспутники. В то же время как для прикладных, так и для фундаментальных исследований необходимо получать изображения солнечной короны в вакуумном ульрафиолетовом (ВУФ) диапазоне спектра. Несмотря на то, что телескопы ВУФ диапазона являются сложным астрокосмическим инструментом, они могут быть адаптированы под размещение на сравнительно небольших наноспутниковых платформах с ограниченными ресурсами. Подобные проекты в настоящее время активно прорабатываются в ведущих космических державах [3].

\section{1. Спектральные диапазоны наблюдения корональной плазмы с помощью космических телескопов}

В настоящее время есть несколько наиболее востребованных для научных и прикладных исследований спектральных диапазонов регистрации короны, которые в первую очередь характеризуются температурой регистрируемой солнечной плазмы. 
Наиболее холодный диапазон плазмы, переходный слой, соответствует температурам 20-100 тыс. градусов. Переходный слой располагается непосредственно над хромосферой Солнца и характеризуется малой толщиной. Именно в нем происходит разогрев плазмы до корональных температур. Для решения фундаментальных задач солнечной физики наблюдения этого слоя интересны с точки зрения транспорта энергии и материи их фотосферы в корону, механизма нагрева плазмы и структуры и динамики „магнитного коврика“. С точки зрения прогнозирования космической погоды переходный слой важен по двум причинам: в нем хорошо локализуются корональные дыры - области, которые являются источником высокоскоростного солнечного ветра, и протуберанцы, разрушение которых может приводить к эруптивным процессам на Солнце. Наблюдение переходного слоя традиционно проводится в линии HeII с длиной волны $30.4 \mathrm{~nm}$ (температура возбуждения 50-70 тыс. градусов).

Второй температурный слой - это собственно корона Солнца с температурой от 500 тыс. до 2 млн. градусов. Плазма с такой температурой занимает большую часть короны. Она довольно хорошо структурирована, в ней хорошо локализованы основные корональные структуры: активные области, силовые трубки магнитного поля, корональные дыры и пр. Эруптивные выбросы коронального вещества так же состоят в основном из этой плазмы. Для фундаментальной физики Солнца и прикладных задач наиболее интересны вспышечные процессы, связанные с концентрацией энергии в плазме этого температурного диапазона, и динамика эруптивных процессов. Как правило, наблюдение этой плазмы проводится в линиях FeIX-FeXIII - наиболее интенсивных после НеII линиях ВУФ области спектра солнечной короны. При этом самая интенсивная линия из вышеназванных - FeIX $17.1 \mathrm{~nm}$.

Третий температурный слой - это вспышечная плазма с температурами выше 3 млн. градусов. Она практически отсутствует в невозмущенной короне и появляется только во время вспышечных процессов, локализованных в небольших областях. Несмотря на короткое время существования этой плазмы и локализацию областей, где она возникает, ее наблюдения крайне важны для понимания природы вспышек и прогноза их развития. Наблюдается вспышечная плазма в линиях ионов с высокой степенью ионизации, например MgXII, Fe XXII-XXV. При этом почти все „горячие“ линии в корональной плазме сильно блендированы более холодными линиями. По этой причине наиболее популярный диапазон для наблюдения вспышек - вблизи $13.2 \mathrm{~nm}$, где сконцентрировано несколько интенсивных линий FeXXIII-FeXIV, a корональные линии довольно слабые.

Таким образом, как с точки зрения прогнозирования космической погоды, так и для решения задач фундаментальной физики наблюдения короны Солнца необходимо проводить в нескольких спектральных диапазонах, соответствующих различным температурным слоям.

\section{2. Наноспутник „Норби-2“}

В рамках программы запуска малых космических аппаратов „УниверСат“ Госкорпорации „Роскосмос“ [4] в конце 2021 г. запланирован запуск наноспутника (малого космического аппарата, МКА) „Норби-2“ с солнечным ВУФ телескопом. „Норби-2“ разрабатывается на основе наноспутника „Норби“ [5], успешно запущенного на низкую околоземную орбиту в сентябре 2020 г. и проходящего в настоящее время летные испытания. Наноспутник „Норби-2“ формата $6 \mathrm{U}(300 \times 200 \times 100 \mathrm{~mm})$, так же как и „Норби“, создается на базе платформы сверхмалых космических аппаратов Новосибирского государственного университета, совместимой со стандартом CubeSat. Базовыми подсистемами платформы являются:

- система электроснабжения СЭС;

- бортовой радиокомплекс БРК с функциями бортовой системы управления;

- система ориентации и позиционирования СОП;

- модуль сопряжения с полезной нагрузкой.

Система электроснабжения СЭС обеспечивает электропитанием все подсистемы наноспутника. Задачами СЭС являются преобразование солнечной энергии в электрическую, накопление энергии и распределение между подсистемами МКА, согласно заданному алгоритму, а также мониторинг текущего состояния параметров системы. Максимальная мощность, генерируемая СЭС, составляет $30 \mathrm{~W}$. Летные испытания „Норби“ на орбите Земли подтвердили расчетные характеристики СЭС и заложенную в нее логику функционирования [5], в связи с чем „Норби-2“ будет укомплектован аналогичной системой электроснабжения без каких-либо существенных изменений.

Основными задачами бортового радиокомплекса „Норби“ с функциями бортовой системы управления являются:

- организация радиосвязи МКА с наземным комплексом управления НКУ;

- управление подсистемами МКА и полезной нагрузкой по автономным алгоритмам или по командам, получаемым от НКУ;

- cбор данных от подсистем МКА и полезной нагрузки и их передача по радиоканалу в НКУ;

- мониторинг состояния подсистем МКА и полезной нагрузки и передача информации об их состоянии в НКУ.

Бортовой радиокомплекс „Норби“ работает на несущей частоте $436.7 \mathrm{MHz}$. Излучаемая мощность передатчика БРК регулируется в диапазоне от 0.1 до $4 \mathrm{~W}$. Отличительной особенностью БРК „Норби“ является применение в радиоканале связи наряду с традиционной частотной манипуляцией FSK широкополосной модуляции LoRa [5,6], разработанной первоначально для сетей Интернета вещей [7]. Модуляция LoRa позволяет значительно увеличить дальность связи или уменьшить мощность передачи, тем самым снижая энергопотребление передатчика. В спутниковой радиосвязи модуляция LoRa применена впервые на „Норби“ . При мощности 
передатчика $0.2 \mathrm{~W}$ и использовании модуляции LoRa БРК „Норби“ обеспечивает передачу данных с орбиты высотой $580 \mathrm{~km}$ на Землю со скоростью до $\sim 10 \mathrm{kbit} / \mathrm{s}$. Максимальная скорость передачи при мощности передатчика $4 \mathrm{~W}$ составляет $\sim 38 \mathrm{kbit} / \mathrm{s}$. Для передачи большого количества изображений с солнечного ВУФ телескопа этого недостаточно. Поэтому для увеличения пропускной способности канала связи „спутник-Земля“ планируется в бортовой радиокомплекс „Норби- $2^{“}$ добавить модуль передатчика мощностью $8 \mathrm{~W}$ на частоте $\sim 2.4 \mathrm{GHz}$. В этом случае при соответствующей модернизации антенного комплекса НКУ и использовании модуляции LoRa скорость передачи по радиоканалу будет достигать $1 \mathrm{Mbit} / \mathrm{s}$ для орбит высотой до $\sim 600 \mathrm{~km}$ при всех углах видимости спутника.

В системе ориентации и позиционирования СОП наноспутника „Норби“ в качестве датчиков ориентации используются датчики направлений на Солнца и Землю, трехосевые магнитометр, датчик угловой скорости и акселерометр, а также приемник ГЛОНАСС $[5,8]$. Все датчики интегрированы в один миниатюрный модуль ориентации датчик Солнце-горизонт ДСГ, всего на поверхности „Норби“ расположено 10 модулей ДСГ. Для стабилизации положения спутника используется магнитная система управления, в которой исполнительным органом управления является система из трех магнитных катушек, ориентированных вдоль главных осей инерции наноспутника. Эта достаточно простая магнитная система управления позволяет решать задачу успокоения наноспутника после отделения от ракетыносителя и ориентировать его относительно Солнца и Земли. Точность определения направления на Солнце датчиком ДСГ составляет $\pm 0.15^{\circ}$, а угловое разрешение $\sim 0.01^{\circ}$ [8]. Магнитная система управления „Норби“ в совокупности с датчиком ДСГ позволяет сориентировать спутник относительно Солнца с точностью $\sim 0.5^{\circ}$. Для повышения точности стабилизации спутника, необходимой для функционирования солнечного ВУФ телескопа, в СОП „Норби-2“ будет добавлена в качестве исполнительного органа система двигателей-маховиков [9]. Типичная точность поддержания ориентации МКА класса CubeSat такими системами составляет $\sim 0.001^{\circ}$ [10].

Модуль сопряжения с полезной нагрузкой представляет собой унифицированный интерфейсный модуль для подключения произвольных полезных нагрузок к платформе МКА.

\section{3. Оптическая схема телескопа ВУФ диапазона для наноспутников}

Выбор оптической схемы телескопа обусловлен сравнительно небольшим объемом, выделяемом на наноспутнике под полезную нагрузку. Учитывая значительную энергоемкость телескопа, необходимость сравнительно мощного передатчика для сброса на Землю получаемой

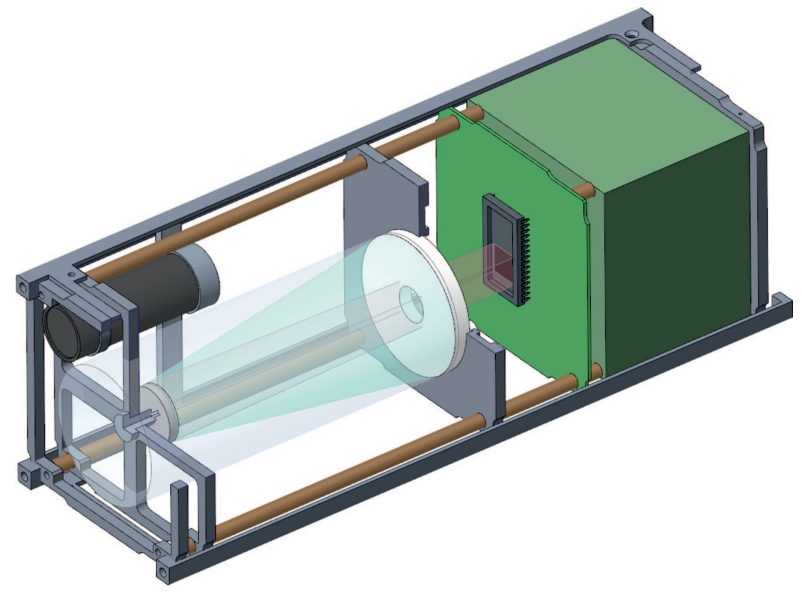

Рис. 1. Компоновка телескопа с двухзеркальной схемой.

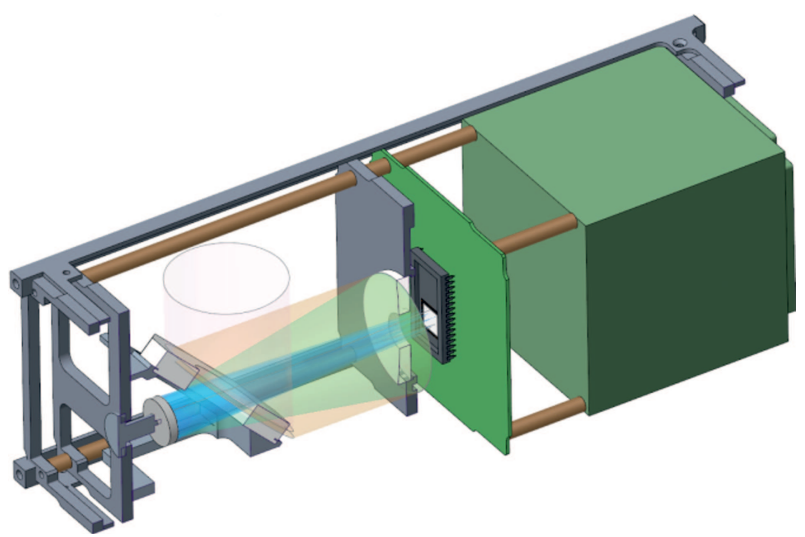

Рис. 2. Компоновка телескопа с трехзеркальной схемой.

информации, наличие относительно прецизионных систем ориентации и стабилизации, размещение телескопа на кубсате возможно только для наноспутника форматом более $6 \mathrm{U}(1 \mathrm{U} \sim 100 \times 100 \times 100 \mathrm{~mm})$. При этом максимальный размер по любой оси не может превышать $300 \mathrm{~mm}$, т. е. для наноспутника габариты 6U составляют $\sim 300 \times 200 \times 100 \mathrm{~mm}$ [11]. С учетом размещения служебных систем спутника под телескоп может быть выделено $3 \mathrm{U}$, т.е. объем $300 \times 100 \times 100 \mathrm{~mm}$. В этом объеме помимо оптических элементов должны располагаться электронные и механические модули телескопа. Исходя из этих ограничений была рассчитана компактная оптическая двухзеркальная система, представляющая собой модернизированную систему Ричи-Кретьена. Компоновка телескопа в такой конфигурации показана на рис. 1.

Недостатком этой схемы с точки зрения функционирования наноспутника является сравнительно небольшое число освещенных солнечных панелей, они занимают площадь $1 \mathrm{dm}^{2}$. В случае выведения входного окна телескопа на „боковую“ сторону телескопа эта площадь возрастает до $5 \mathrm{dm}^{2}$. Поэтому была также рассмотре- 
Таблица 1. Основные характеристики CMOS-матрицы

\begin{tabular}{l|c}
\hline \multicolumn{1}{c|}{ Показатели } & Значения \\
\hline Формат & $2048 \times 2048$ \\
Размер ячейки, $\mu \mathrm{m}$ & $6.5 \times 6.5$ \\
Размер чувствительной области, $\mathrm{mm}$ & $13.3 \times 133$ \\
Тип электронного затвора & перекатывающийся
\end{tabular}

Таблица 2. Основные оптические характеристики телескопов

\begin{tabular}{c|c|c}
\hline \multicolumn{1}{c|}{ Показатели } & $\begin{array}{c}\text { Двухзеркальная } \\
\text { схема }\end{array}$ & $\begin{array}{c}\text { Трехзеркальная } \\
\text { схема }\end{array}$ \\
\hline Поле зрения, $^{\circ}$ & 1 & 1.4 \\
\hline $\begin{array}{l}\text { Разрешение в центре } \\
\text { поля зрения, arc. sec. }\end{array}$ & 0.41 & 1.9 \\
\hline $\begin{array}{l}\text { Разрешение на краю } \\
\text { поля зрения, arc. sec. }\end{array}$ & 2.2 & 2.56 \\
\hline Виньетирование, \% & 43 & 40
\end{tabular}

на схема с ,ломающим“ плоским зеркалом на входе (рис. 2).

Основными недостатками трехзеркальной схемы являются: увеличение веса телескопа, меньшая в 5-10 раз в зависимости от спектрального диапазона чувствительность, более высокие требования к взаимному расположению оптических элементов.

В обоих оптических схемах планируется применение CMOS-матрицы с обратной засветкой GSENSE2020BSIPSкомпании GPIXEL (табл. 1). Особенностью этой матрицы является минимальная толщина технологических и защитных слоев оксида и нитрида кремния, что существенно повышает эффективность регистрации в ВУФ диапазоне спектра.

Характеристики обоих схем с использованием приемника на основе GSENSE2020BSI-PSприведены на табл. 2

\section{4. Многослойные зеркала и фильтры ВУФ диапазона для телескопа}

Для регистрации линий ионов вспышечной плазмы в окрестности длины волны $13.2 \mathrm{~nm}$ эффективны оптические системы на основе многослойных зеркал $\mathrm{Mo} / \mathrm{Si}$, традиционно использующихся в этом диапазоне. Их пиковый коэффициент отражения $R$ может достигать $68 \%$ [12]. При этом величина $\Delta \lambda$ (спектральная ширина пика отражения на половине высоты) составляет $0.53 \mathrm{~nm}$. Этот параметр влияет на спектральное разрешение телескопа.

В работах $[13,14]$ показано, что при необходимости уменьшить величину $\Delta \lambda$ (увеличить спектральное разрешение телескопа) можно путем уменьшения отно- сительной доли молибдена в периоде зеркала. Однако это сопровождается уменьшением $R$. В частности, при $\Delta \lambda=0.35 \mathrm{~nm} R=53 \%$.

Немаловажным преимуществом зеркал $\mathrm{Mo} / \mathrm{Si}$ является высокая временная стабильность их отражательных характеристик. При длительном хранении и использовании, если отсутствуют загрязняющие поверхность факторы, $R$ и $\Delta \lambda$ остаются на прежнем уровне в течение лет.

На основе этой же пары материалов ранее создавались многослойные зеркала для изучения корональной плазмы на длине волны $17.1 \mathrm{~nm}$. Например, зеркала телескопа ТЕРЕК [15] или телескопа ЕІT обсерватории SOHO [16].Однако их отличали относительно невысокие $R$ (до 29\%) и значительная величина $\Delta \lambda$ (около $1.2 \mathrm{~nm}$ ). Оптимизированные позднее $\mathrm{Mo} / \mathrm{Si}$-зеркала имели $R=54 \%, \Delta \lambda=0.875 \mathrm{~nm}[17]$.

Для лучшего сочетания высокого отражения и спектральной селективности необходим переход к зеркалам на основе алюминия, одного из наиболее прозрачных материалов в диапазоне длин волн, больших $17.04 \mathrm{~nm}$ ( $L$-край поглощения алюминия). Такие структуры действительно позволили улучшить спектральное разрешение практически без потери отражательной способности. Так, структуры $\mathrm{Mo} / \mathrm{Al} / \mathrm{B}_{4} \mathrm{C}$ имеют $R=55.5 \%, \Delta \lambda=0.875 \mathrm{~nm}$, a $\mathrm{Mo} / \mathrm{Al} / \mathrm{SiC}-R=53.4 \%$, $\Delta \lambda=0.76 \mathrm{~nm}[18,19]$. Лучше разрешение при сопоставимом коэффициенте отражения имеет $\mathrm{Zr} / \mathrm{Al}: R=56 \%$, $\Delta \lambda=0.6 \mathrm{~nm}[20]$.

Еще больший выигрыш дает стратегия применения для создания зеркал двух относительно слабо поглощающих материалов. В работе [21] в качестве таких материалов предложены алюминий и бериллий. Оба материала имеют малое поглощение в рассматриваемом диапазоне длин волн, однако при этом между ними сохраняется значительный оптический контраст (разница показателей преломления). Там же для снижения межслоевой шероховатости между $\mathrm{Al}$ и $\mathrm{Be}$ предложено использовать аморфизующее свойство тонкого барьерного слоя кремния. В итоге получилось зеркало $\mathrm{Al} / \mathrm{Be} / \mathrm{Si}$ с $R=61 \%$ и $\Delta \lambda=0.4 \mathrm{~nm}$.

Мы провели оптимизацию параметров структуры и получили зеркало, спектральная зависимость коэффициента отражения которого приведена рис. 3. Пиковый коэффициент отражения на длине волны $17.1 \mathrm{~nm}$ составил $62.5 \%, \Delta \lambda=0.3 \mathrm{~nm}$.

Этот же состав $(\mathrm{Al} / \mathrm{Be} / \mathrm{Si})$ целесообразно использовать для синтеза зеркал, предназначенных для изучения переходного слоя на длине волны $30.4 \mathrm{~nm}$. В работе [22] показано, что структуры $\mathrm{Al} / \mathrm{Be} / \mathrm{Si}$ обладают наилучшим сочетанием (для этого диапазона) коэффициента отражения и спектральной селективности: $R=34.3 \%$ и $\Delta \lambda=1 \mathrm{~nm}$.

В работе [14] изучалась временная стабильность отражательных характеристик многослойных структур $\mathrm{Al} / \mathrm{Be} / \mathrm{Si}$. Показано, что в течение, по крайней мере, 20 месяцев не наблюдается их ухудшения. 


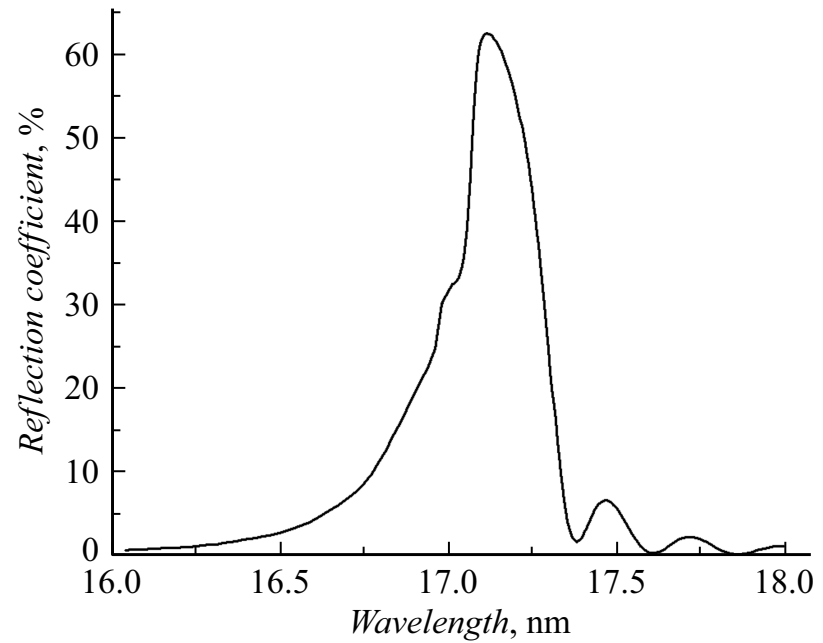

Pис. 3. Спектральная зависимость коэффициента отражения зеркала $\mathrm{Al} / \mathrm{Be} / \mathrm{Si}$. Угол падения излучения $2^{\circ}$ от нормали. Измерения выполнены на синхротроне BESSY-II.

Таблица 3. Характеристики отражающих покрытий многослойных зеркал

\begin{tabular}{c|c|l|l}
\hline Канал регистрации & Структура & $R, \%$ & $\Delta \lambda, \mathrm{nm}$ \\
\hline $13.2 \mathrm{~nm}$ & Mo/Si с максимальным $R$ & 68 & 0.53 \\
$13.2 \mathrm{~nm}$ & $\mathrm{Mo} / \mathrm{Si}$ узкополосная & 53 & 0.35 \\
$17.1 \mathrm{~nm}$ & $\mathrm{Al} / \mathrm{Be} / \mathrm{Si}$ & 62.5 & 0.3 \\
$30.4 \mathrm{~nm}$ & $\mathrm{Al} / \mathrm{Be} / \mathrm{Si}$ & 34.3 & 1
\end{tabular}

В табл. 3 представлены многослойные структуры, которые предлагается использовать в качестве отражающих покрытий оптических элементов солнечной обсерватории. Пленочные абсорбционные фильтры традиционно применяются в солнечных телескопах экстремального ультрафиолетового (ЭУФ) диапазона для подавления излучения Солнца в видимом и инфракрасном (ИК) диапазоне. Выбирая состав фильтра из материалов, имеющих низкое поглощение в рабочем спектральном диапазоне телескопа, можно добиться того, чтобы фильтр пропускал излучение в ЭУФ диапазоне и блокировал более длинноволновое. Естественно, что стремятся так оптимизировать состав и толщину пленочного фильтра, чтобы он пропускал как можно больше полезного сигнала в рабочем диапазоне и одновременно обеспечивал требуемую степень подавления излучения видимого и ИК диапазонов.

Для перекрытия спектрального диапазона $13-35 \mathrm{~nm}$ в обсерватории ТЕСИС [23] применялась взаимодополняющая пара многослойных абсорбционных фильтров $\mathrm{Zr} / \mathrm{Si}$ и $\mathrm{Al} / \mathrm{Si}$. Состав и структура пленочных фильтров обеспечивали требуемый уровень подавления света (лучше, чем $10^{6}$ в видимом диапазоне) и высокую механическую прочность, необходимую чтобы пленочные фильтры выдержали без повреждения вывод спутника с телескопами на орбиту. Уровень подавления излучения в видимом и ИК диапазонах определяется в основном толщиной металла в фильтре, а кремний вносит дополнительное поглощение в ЭУФ диапазоне (особенно, в длинноволновой его части). Отсюда очевидно, что большего коэффициента пропускания в диапазоне $17-35 \mathrm{~nm}$ при сохранении уровня подавления видимого и ИК излучения можно добиться с использованием однородной алюминиевой пленки. Однако однокомпонентный алюминиевый фильтр менее прочен, чем $\mathrm{Al} / \mathrm{Si}$. Кроме того, алюминий со временем способен окисляться на значительную толщину, что нежелательно, так как это снижает его коэффициент пропускания в ЭУФ диапазоне.

В более поздней работе [24] для повышения прочности и стойкости к окислению Al-фильтров было предложено использовать защитные покрытия $\mathrm{MoSi}_{2}$ нанометровых толщин. Так как покрытия тонкие, то они вносят заметно меньший вклад в поглощение в ЭУФ диапазоне, чем в случае использования $\mathrm{Si-прослоек,}$ а потому при сравнимых оптических и механических характеристиках $\mathrm{MoSi} 2 / \mathrm{Al} / \mathrm{MoSi}_{2}$-фильтры превосходят по коэффициенту пропускания использовавшиеся ранее многослойные $(\mathrm{Al} / \mathrm{Si})$ фильтры. В случае $\mathrm{Zr} / \mathrm{Si}$-фильтров вклад кремния в снижение коэффициента пропускания не столь значителен (из-за близости полосы пропускания $\mathrm{Zr} / \mathrm{Si}$-фильтра к $L$ краю поглощения $\mathrm{Si}$ ).

Таким образом, в данном проекте предлагается использовать $\mathrm{Al}$ и многослойные $\mathrm{Zr} / \mathrm{Si}$-фильтры. Для повышения уровня защиты от окисления предлагается использовать защитные покрытия из $\mathrm{MoSi}_{2}$, также и в случае $\mathrm{Zr} / \mathrm{Si}$-многослойного фильтра.

На рис. 4 приведены расчетные коэффициенты пропускания для следующих пленочных фильтров:

1) $\mathrm{MoSi}_{2}-3 \mathrm{~nm}, \mathrm{Al}-150 \mathrm{~nm}, \mathrm{MoSi}_{2}-3 \mathrm{~nm}$;

2) $\mathrm{MoSi}_{2}-3 \mathrm{~nm},(\mathrm{Zr}-3 \mathrm{~nm} / \mathrm{Si}-1 \mathrm{~nm}) \cdot 50, \mathrm{MoSi}_{2}-$ $3 \mathrm{~nm}$.

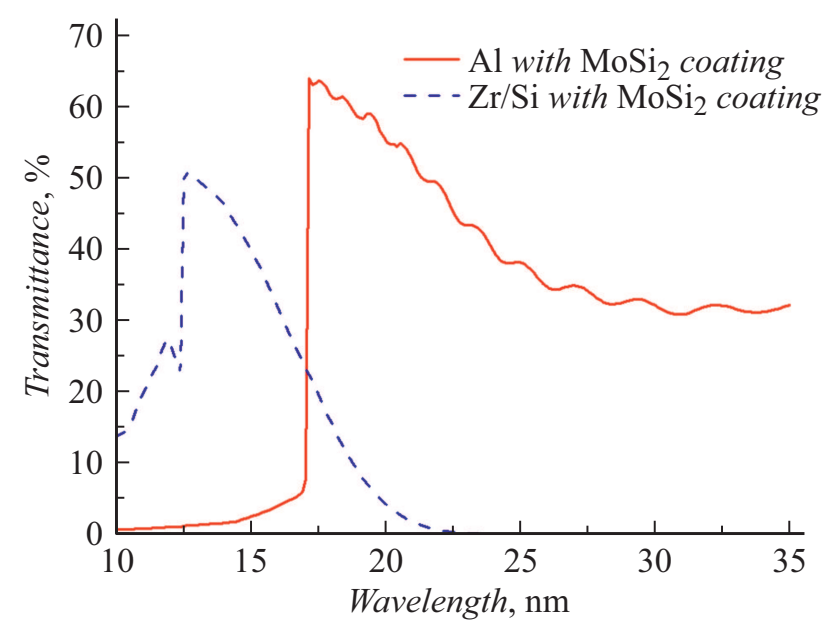

Рис. 4. Расчетные спектры пропускания $\mathrm{Zr} / \mathrm{Si}$ и $\mathrm{Al}$ пленочных фильтров с защитными $\mathrm{MoSi}_{2}$-покрытиями при нормальном падении. При расчетах использовались оптические константы, взятые из базы данных CXRO [25]. 
Расчетные коэффициенты пропускания пленочных фильтров на длинах волн, соответствующих каналам регистрации телескопов, составляют: $T(13.2 \mathrm{~nm})=49 \%$, $T(17.1 \mathrm{~nm})=63 \%, T(30.4 \mathrm{~nm})=31 \%$.

Выбранные толщины пленок обеспечивают требуемый уровень подавления видимого света (лучше, чем $10^{6}$ ).

\section{Заключение}

Продемонстрирована возможность создания миниатюрных телескопов для получения ВУФ изображений короны Солнца для наноспутников. Отработку этих инструментов предполагается провести в рамках программы Роскосмоса „Универсат“ в 2021-2025гг. Ближайший запуска КА „Норби“ с ВУФ телескопом на диапазон $17.1 \mathrm{~nm}$ запланирован на конец 2021 г.

\section{Финансирование работы}

Работа выполнена при поддержке Министерства науки и высшего образования в рамках выполнения работ по Государственному заданию ФНИЦ „Кристаллография и фотоника“ РАН, ФИАН, ИСЗФ СО РАН и ИФМ РАН.

Работа частично выполнена в рамках проекта FSSS-2020-0018, финансируемого из средств государственного задания победителям конкурса научных лабораторий образовательных организаций высшего образования, подведомственных Минобрнауки России.

\section{Конфликт интересов}

Авторы заявляют, что у них нет конфликта интересов.

\section{Список литературы}

[1] J.P. Mason, T.N. Woods, A. Caspi, P.C. Chamberlin, C. Moore, A. Jones, R. Kohnert, X. Li, S. Palo, S.C. Solomon. J. Spacecraft Rockets, 53 (2), 328 (2016).

[2] Н.В. Гончаров, М.Ю. Корецкий, В.И. Майорова, В.Г. Мельникова, Н.А. Неровный, Д.А. Рачкин, С.М. Тененбаум, Е.Д. Тимакова, К.А. Фролов, И.В. Ястребова, С.А. Богачев, С.Ю. Дятков, А.С. Кириченко, С.В. Кузин, А.А. Перцов. Космонавтика и ракетостроение, 1 (100), 69 (2018).

[3] J.P. Mason, Ph.C. Chamberlin1, D. Seaton J. Burkepile, R. Colaninno, K. Dissauer, F.G. Eparvier, Y. Fan, S. Gibson, A.R. Jones, C. Kay, M. Kirk, R. Kohnert, W.D. Pesnell, B.J. Thompson, A.M. Veronig, M.J. West, D. Windt, T.N. Woods. J. Space Weather Space Clim., 11 (20), (2021).

[4] Электронный ресурс. Режим доступа: https://www.roscosmos.ru/23836/

[5] V.Yu. Prokopyev, S.S. Bakanov, V.K. Bodrov, E.N. Chernodarov, A.A. Doroshkin, V.N. Gorev, A.Yu. Kolesnikova, A.S. Kozlov, O.N. Kus, A.V. Melkov, A.A. Mitrokhin, A.A. Morsin, A.E. Nazarenko, I.V. Neskorodev, A.V. Pelemeshko, Yu.M. Prokopyev, D.A. Romanov, A.M. Shilov, M.V. Shirokih, A.A. Sidorchuk, A.S. Styuf, A.M. Zadorozhny. J. Phys.: Conf. Ser. (2021, in press).
[6] A. Doroshkin, A. Zadorozhny, O. Kus, V. Prokopyev, Yu. Prokopyev. IEEE Access, 7, 75721 (2019). DOI: 10.1109/ACCESS.2019.2919274

[7] C. Goursaud, J.-M. Gorce. EAI Endorsed Trans. Internet Things, 15 (1), e3 (2015). DOI: $10.4108 /$ eai.26-10-2015.150597

[8] A. Pelemeshko, A. Kolesnikova, A. Melkov, V. Prokopyev, A. Zadorozhny. IOP Conf. Ser:: Mater. Sci. Eng., 734, 012012 (2020). DOI: 0.1088/1757-899X/734/1/012012

[9] В.Н. Васильев. Системы ориентащии космических аппаратов (ФГУП „НПП ВНИИЭМ“, М., 2009)

[10] Z. Ismail, R. Varatharajoo. Adv. Space Res., 45, 750 (2010). DOI: $10.1016 /$ j.asr.2009.11.004

[11] CDS. 6UCubeSatDesignSpecification. Rev. Provisional. California Polytechnic State University, 2016.

[12] A.E. Yakshin, R.W.E. van de Kruijs, I. Nedelcu, E. Zoethout, E. Louis, F. Bijkerk, H. Enkisch, S. Müllender. Proc. SPIE, 6517, 65170I (2007). DOI: 10.1117/12.711796

[13] S.A. Bogachev, N.I. Chkhalo, S.V. Kuzin, D.E. Pariev, V.N. Polkovnikov, N.N. Salashchenko, S.V. Shestov, S.Y. Zuev. Appl. Opt., 55 (9), 2126 (2016). doi.org/10.1364/AO.55.002126

[14] N. Chkhalo, A. Lopatin, A. Nechay, D. Pariev, A. Pestov, V. Polkovnikov, N. Salashchenko, F. Schäfers, M. Sertsu, A. Sokolov, M. Svechnikov, N. Tsybin, S. Zuev. J. Nanosci. Nanotechnol., 19, 546 (2019).

[15] A. Ignatiev, N. Kolachevsky, V. Korneev, V. Krutov, S. Kuzin, A. Mitrofanov, A. Pertsov, E. Ragozin, V. Slemzin, I. Tindo, I. Zhitnik. Proc. SPIE, 3406, 20 (1998).

[16] J.-P. Delaboudinière, G.E. Artzner, J. Brunaud, A.H. Gabriel, J.F. Hochedez, F. Millier, X.Y. Song, B. Au, K.P. Dere, R.A. Howard, R. Kreplin, D.J. Michels, J.D. Moses, J.M. Defise, C. Jamar, P. Rochus, J.P. Chauvineau, J.P. Marioge, R.C. Catura, J.R. Lemen, L. Shing, R.A. Stern, J.B. Gurman, W.M. Neupert, A. Maucherat, F. Clette, P. Cugnon, E.L. Van Dessel. Solar Physics, 162 (1-2), 291 (1995).

[17] R. Soufli, D.L. Windt, J.C. Robinson, S.L. Baker, E. Spiller, F.J. Dollar, A.L. Aquila, E.M. Gullikson, B. Kjornrattanawanich, J.F. Seely, L. Golub. Proc. SPIE, 5901, 590101 (2005). DOI: 10.1117/12.617370

[18] M.H. Hu, K. Le Guen, J.M. André, P. Jonnard, E. Meltchakov, F. Delmotte, A. Galtayries. Opt. Express, 18(19), 20019 (2010). DOI: 10.1364/OE.18.020019

[19] E. Meltchakova, A. Ziani, F. Auchere, X. Zhang, M. Roulliay, S. De Rossi, Ch. Bourassin-Bouchet, A. Jérome, F. Bridou, F. Varniere, F. Delmotte. Proc. SPIE, 8168, 816819 (2011). https://doi.org/10.1117/12.896577

[20] С.Ю. Зуев, С.В. Кузин, В.Н. Полковников, Н.Н. Салащенко. Известия РАН. Серия физическая, 74 (1), 58 (2010). [S.Yu. Zuev, V.N. Polkovnikov, N.N. Salashchenko, S.V. Kuzin. Bull. Russ. Acad. Sci.: Phys., 74 (1), 50 (2010).]

[21] N.I. Chkhalo, D.E. Pariev, V.N. Polkovnikov, N.N. Salashchenko, R.A. Shaposhnikov, I.L. Stroulea, M.V. Svechnikov, Yu.A. Vainer, S.Yu. Zuev. Thin Solid Films, 631, 106 (2017). https://doi.org/10.1016/j.tsf.2017.04.020

[22] В.Н. Полковников, Н.Н. Салащенко, М.В. Свечников, Н.И. Чхало. УФН, 190, 92 (2020). DOI: $10.3367 /$ UFNr.2019.05.038623 [V.N. Polkovnikov, N.N. Salashchenko, M.V. Svechnikov, N.I. Chkhalo. Phys. Usp., 63 (1) (2020).] 
[23] S.V. Kuzin, I.A. Zhitnik, S.V. Shestov, S.A. Bogachev, O.I. Bugaenko, A.P. Ignat'ev, A.A. Pertsov, A.S. Ulyanov, A.A. Reva, V.A. Slemzin, N.K. Sukhodrev, Yu.S. Ivanov, L.A. Goncharov, A.V. Mitrofanov, S.G. Popov, T.A. Shergina, V.A. Solov'ev, S.N. Oparin, A.M. Zykov. Sol. Syst. Res., 45, 166 (2011).

[24] N.I. Chkhalo, M.N. Drozdov, S.A. Gusev, A.Ya. Lopatin, V.I. Luchin, N.N. Salashchenko, D.A. Tatarskiy, N.N. Tsybin, S.Yu. Zuev. Appl. Opt., 58 (1), 21 (2019). https://doi.org/10.1364/AO.58.000021 25.

[25] Электронный ресурс. Режим доступа: https://henke.lbl.gov/optical_constants/ 